\title{
EFECTOS DEL ESTATUS Y LA MOVILIDAD SOCIALES SOBRE LA ILEGITIMIDAD* Y EL MATRIMONIO SUBSECUENTE
}

\author{
SUGIYAMA IUTAKA \\ Universidad de Florida
}

\section{INTRODUCCIÓN}

Ciertas características de investigaciones anteriores sobre la ilegitimidad han limitado de modo serio, el grado hasta el cual pudieran generalizarse los hechos encontrados. Por ejemplo, muy pocos estudios empíricos se han basado en muestras representativas de poblaciones conocidas. ${ }^{1}$ Mas aún, han fracasado en la especificación, a cualquier grado de alcance, de la importancia relativa de las variables asociadas con la ilegitimidad. ${ }^{2}$ Por último, con la excepción de los estudios que utilizan materiales demográficos, la mayoría de las investigaciones sobre la ilegitimidad se han restringido a poblaciones de los Estados Unidos. ${ }^{3}$

Los propósitos del presente estudio son: 1) presentar datos obtenidos por una muestra aleatoria de una población conocida; 2) probar ciertos aspectos de la teoría de la ilegitimidad (basada en su mayor parte en poblaciones de los Estados Unidos); y 3) ampliar el conocimiento en esta área mediante la determinación de la significación relativa de los factores asociados con la ilegitimidad.

Pope ha notado que un cierto número de factores interdependientes se relacionan con la probabilidad de que una mujer tenga un hijo ile-

* N. del T. El término en inglés "illegitimacy" significa en el presente artículo "la condición de tener un hijo ilegítimo", sin embargo, y por brevedad, lo tomamos aquí como "ilegitimidad".

1 Vincent ha demostrado de manera efectiva cómo estudios anteriores se han basado en nuestras sesgadas. Véase Clark E. Vincent, "The Unwed Mother and Sampling Bias", American Sociological Review, Vol. 19, Núm. 5, octubre de 1954, pp. 562-567. En apariencia, Pope ha presentado el único estudio que utiliza muestreo de algún universo conocido. Véase Hallowell Pope, "Unwed Mothers and Their Sex Partners", Journal of Marriage and the Family, Vol. 29, Núm. 3, agosto de 1967, pp. 557-567.

2 Véase Jane Collier Kronick, "An Assesment of Research Knowledge Concerning the Unmarried Mother", en la edición de Robert W. Roberts, The Unwed Mother, Nueva York, Harper and Row, 1966, pp. 242-244 y 250, sobretiro de Research Perspectives on the Unmarried Mother, editado por Child Welfare League of America, Nueva York, 1962, pp. 17-31.

3 Para algunas excepciones, véase Judith Blake, et al., Family Structure in Jamaica, Glencoe, Free Press, 1961; y J. Mayone Stycos y Kutr W. Back, The Control of Human Fertility in Jamaica, Ithaca, Cornell University Press, 1964. 
gítimo. ${ }^{4}$ Muchos de estos factores (por ejemplo, las actitudes hacia relaciones sexuales premaritales, los anticonceptivos y el aborto) varían entre las subpoblaciones de cualquier sociedad dada. Falta que se determine si estas variaciones de deben a puntos de vista diferenciales del "principio de la legitimidad" o son reflejos de diferencias en las normas observadas por las subpoblaciones. ${ }^{5}$ Sin embargo, estas variaciones sí ayudan a producir tasas diferenciales de ilegitimidad entre las distintas categorías raciales, de edad, y de clase social, en los Estados Unidos.

Aunque la investigación es limitada, se ha encontrado que cierto número de factores se encuentran repetidamente asociados con la ilegitimidad: edad, raza, clase social (medida por la ocupación, educación e ingreso), y la religión. ${ }^{6}$ Se ha encontrado que todas estas variables fundamentales tambiên están relacionadas con factores que afectan más directamente la probabilidad de una maternidad sin matrimonio. Por ejemplo, factores tales como relaciones sexuales prematrimoniales, ${ }^{7}$ uso de anticonceptivos, ${ }^{8}$ embarazo premarital, ${ }^{9}$ aborto, ${ }^{10}$ tipo de relación con el padre putativo, ${ }^{11}$ y el matrimonio antes del nacimiento del hijo, ${ }^{12}$ han demostrado mantener una relación con los indicadores de clase social. Así, en comparación con una pareja de clase media o alta, es más probable que las parejas provenientes de clases más bajas experimenten relaciones sexuales durante el noviazgo, menos probable que utilicen anticonceptivos, más probable que se vean envueltas en un embarazo prematrimonial, y menos probable que realicen un aborto. También es más factible que conserven al niño en lugar de cederlo para su adopción. ${ }^{13}$ De este modo,

4 Pope, op. cit. p. 555.

5 Goode sostiene que las diferencias en las tasas de ilegitimidad se deben a diferencias en el compromiso con las normas sociales. Véase William J. Goode, "Illegitimacy in the Caribbean Social Structure", American Sociological Review, Vol. 25, Núm. 1, febrero de 1960, p. 27. Los escritos de otros autores parecen implicar que las tasas diferenciales son resultado de las diferentes normas adoptadas por las distintas subpoblaciones. Véase por ejemplo, Kronick, op. cit., pp. 242-244. Vincent indica que se involucran tanto diferentes compromisos sociales como "contra normas". Véase Clark E. Vincent, Unmarried Mothers, Glencoe, Free Press, 1961, pp. 5-17.

6 Véase por ejemplo, Paul H. Gebhard, et al., Pregnancy, Birth and Abortion, Nueva York, Harper and Brothers, 1958, relato 28, p. 78.

$\tau$ Véase por ejemplo Alfred C. Kinsey, et al., Sexual Behavior in the Human Male, Philadelphia, W. B. Saunders Co., 1948, pp. 430-431 y Alfred C. Kinsey et al., Sexual Behavior in the Human Female, Philadelphia, W. B. Saunders Co., 1953, p. 333.

8 Lee Rainwater y Karol Weinstein, And the Poor Get Children, Chicago, Quadrangle Books, 1960, Capítulos 7 y 8; Pope, op. cit., pie de página 8, p. 563.

9 Harold T. Christensen, "Cultural Relativism and Premarital Sex Norms", American Sociological Review, Vol. 25, Núm. 1, febrero de 1960, p. 34.

10 Gebhard, et al., loc. cit.

11 Clark E. Vincent, "Ego Involvement in Sexual Relations: Implications for Research on Illegitimacy", American Journal of Sociology, Vol. 65, Núm. 3.

12 Gebhard, et al., loc. cit.

13 Henry J. Meyer, et al., "The Decision by Unmarried Mothers to Keep or Surrender their Babies", Social Work, Vol. 1. Núm. 2, abril de 1956, pp. 103-109; Henry J. Meyer, et al., "Unwed Mothers' Decisions About their Babies: An Interim Replication Study", Child Welfare, Vol. 38, Núm. 2, febrero de 1959, pp. 1-6; y Wyatt C. Jones, et al., "Social and Psychological Factors in Status Decision of 
es más plausible que las mujeres de clase baja se conviertan en madres solteras y que conserven a sus hijos, que aquellas mujeres de clase media y alta. En resumen, todo un juego de comportamiento en el noviazgo, y no sólo la ilegitimidad, es variable de acuerdo con la condición socioeconómica. La ilegitimidad puede parecer un resultado "normal" de un proceso "normal" de subcultura. Así, por ejemplo, Pope concluye que, mientras que un nacimiento premarital puede tomarse como un extravío, el tipo de noviazgo que lleva a tal acontecimiento generalmente no difiere de otra clase de cortejo. ${ }^{14}$

Aunque se ha encontrado que los factores de fondo mencionados anteriormente tienen que ver con la ilegitimidad, no obstante tanto su importancia relativa como su interacción sobre la probabilidad de este fenómeno, no han sido firmemente establecidas todavía. Hasta el momento, el mejor indicador es el de que la condición socio-económica es uno de los factores más importantes relacionado tanto con la conducta del noviazgo como con sus resultados: la presencia o ausencia de un nacimiento ilegítimo. Aunque las diferencias en la ilegitimidad entre clases sociales están desapareciendo en los Estados Unidos, ${ }^{15}$ la condición económica parece ser aún en muchas formas la variable más importante estudiada hasta la fecha. ${ }^{16}$

El estudio presente investiga algunos factores sociales alrededor de la ilegitimidad, e intenta determinar las interacciones e importancia relativa de éstos. También se estudian algunos factores sobre un aspecto des. cuidado de la ilegitimidad: el matrimonio subsecuente. De este modo, la investigación puede verse en parte como una ampliación de resultados anteriores.

A la luz de tales trabajos, se formulan las siguientes proposiciones:

1) En comparación con las mujeres que no tienen un hijo ilegítimo, para aquellas que sí lo tienen es más factible que $a$ ) se casen a una edad temprana; b) pertenezcan a una religión no católica; $c$ ) participen sólo marginalmente, o se separen totalmente de organizaciones religiosas formales; d) hayan tenido un nivel educativo bajo; y $e$ ) provengan de una clase baja;

2) De todas las variables en consideración, la condición social es el factor discriminatorio más importante entre las mujeres que tienen y las que no tienen hijos ilegítimos;

3) Aquellas mujeres que no muestran movilidad social alguna, están más expuestas a tener un hijo ilegítimo que aquellas que denotan un ascenso social, pero menos expuestas que las que tienen un descenso social.

En apariencia no existen investigaciones respecto al matrimonio de mujeres que han tenido un hijo ilegítimo. Aunque a fin de cuentas la mayoría de las mujeres se casen, cierto número de factores afectan el lapso entre la maternidad en estado de soltera y el matrimonio subsecuente.

Unmarried Mothers", Marriage and Family Living, Vol. 24, Núm. 3, agosto de 1962, pp. 224-230. Véase también Vincent, Unmarried Mothers, op. cit., p. 188.

14 Pope, op. cit., p. 567.

15 Robert W. Roberts (Comp.), The Unwed Mother, Nueva York, Harper and Row, 1966, p. 4.

16 Cf. Kronick, op. cit., pp. 242-244 y 250. 
El presente estudio hizo posible la formulación de las proposiciones siguientes.

4) El lapso entre la maternidad sin matrimonio y el casamiento posterior es más corto que aquellas mujeres que: a) se casan a edades jóvenes; b) son católicas; c) participan en organizaciones religiosas; d) han alcanzado un alto nivel de educación; y e) son de un estatus socio-económico alto;

5) De todos los factores bajo consideración, el estatus social es el factor discriminatorio más significativo entre aquellas que se casan más pronto y las que contraen matrimonio más tardiamente después del nacimiento del hijo ilegítimo;

6) Las mujeres que no muestran movilidad social alguna toman más tiempo para casarse después de tener un hijo natural, que aquellas que ascienden socialmente, pero a su vez requieren un lapso menor que las que muestran un descenso.

\section{Metodología}

Los datos utilizados en este trabajo fueron recolectados de un estudio de fertilidad y planeación familiar realizado en Río de Janeiro, Brasil, en $1963 .{ }^{17}$ Se usó un muestreo por área para escoger 2496 mujeres que representaran la población femenina de esa ciudad. Se tomaron mujeres entre los 20 y los 50 años de edad sin importar su estado civil.

Varias consideraciones redujeron el número de mujeres utilizadas en el estudio. Se excluyeron todas las que no habían tenido al menos un hijo nacido vivo dado que el objetivo del estudio era investigar los factores relacionados con la ilegitimidad en las mujeres que han tenido hijos, más que describir las tasas de ilegitimidad de la población total. Esta consideración redujo la muestra inicial a 1834 mujeres. De este último total, 117 o sea el $6.4 \%$ no se incluyó en el análisis porque faltaba información sobre la fecha de matrimonio o la fecha del primer nacimiento vivo, datos esenciales para el cómputo del intervalo de tiempo entre el nacimiento ilegítimo y el matrimonio subsecuente. Tomando en cuenta que el $6.4 \%$ de información incompleta constituye una tasa relativamente alta, se da una breve caracterización de estas mujeres. ${ }^{18} \mathrm{E} 1$

17 Esta investigación es parte de "Siete encuestas sobre fertilidad y planeación familiar", dirigida por el Centro Latinoamericano de Demografía, Santiago, Chile. El estudio fue financiado por el Consejo de la Población e instituciones locales llevaron a cabo su propio estudio nacional. La encuesta en Río de Janeiro fue llevada a cabo por el Centro Latinoamericano de Investigación en Ciencias Sociales y fue dirigida por Sugiyanıa Iutaka mientras era miembro de esa institución. Para mayores detalles véase: León Tabah, "Plan de recherche de sept enquetes comparatives sur la fecondite en Amerique Latine", Population, París, Vol. 19, Núm. 1, 1964, pp. 95-126.

18 Obtener datos completos ha sido uno de los problemas más comunes con los que se han enfrentado los investigadores que llevan a cabo estudios cuantitativos en países en desarrollo. Véase Sugiyama Iutaka, "Investigaciones comparativas en áreas urbanas: problemas y perspectivas de estudio en América Latina", Sociología y Sociedad en Latinoamérica, Bogotá, Asociación Colombiana de Sociología, 1966, pp. 9-23. 
bajo nivel educativo de la población puede explicar parcialmente este fenómeno, al igual que la falta de experiencia en la recolección de información mediante este tipo de instrumento. De aquellas mujeres que en teoría estarían en posibilidad de dar información completa (o sea las casadas una vez), $71.0 \%$ no completaron ni siquiera cuatro años de educación formal. Más aún, se encontró que $52.6 \%$ de estas mujeres con menos de cuatro años de educación formal eran de 40 años de edad y más. En resumen, la falta de información redujo el total en la muestra a 1625 .

La ilegitimidad se define para aquellas mujeres que tuvieron un hijo antes de casarse. Solamente primeros matrimonios y primeros hijos se tomaron en cuenta en el estudio. La relación matrimonial incluye relaciones legales y consensuales. En ambos casos se pidió a las encuestadas que dieran las fechas de comienzo del matrimonio o unión.

Otras mediciones usadas en esta investigación y que necesitan explicarse son: educación, condición social, religiosidad, y movilidad social. La educación se divide en tres categorías: ninguna, elemental (hasta cuatro años), y secundaria (seis años o más). ${ }^{9}$ El estatus social de las mujeres se asimiló a éstas utilizando la ocupación de los maridos y los padres. La jerarquía de las categorías de estatus (medida por la ocupación), fue verificada por Hutchinson para el caso brasileño. ${ }^{20}$ Las seis categorías resultantes se agruparon primero en tres estratos y después en los niveles de ocupaciones manuales y no manuales. Las dos categorías más bajas de las seis constituyeron el estrato de ocupaciones manuales. En los católicos la religiosidad se midió por la frecuencia de asistencia a las iglesias. Aquellos que van a la iglesia al menos tres veces al mes y comulgan al menos una vez al año, se clasificaron como católicos; los que declararon ser católicos pero que no asisten a culto religioso alguno se clasificaron como católicos no practicantes; todos los demás se tomaron como católicos semipracticantes. Por último, la movilidad social se consideró de tres maneras diferentes: 1) la presencia o ausencia de movilidad social; 2) el sentido de la movilidad social; y 3) el sentido de la movilidad social tomando en cuenta los antecedentes del estatus social de las mujeres. Para las dos primeras medidas de movilidad social se tomaron en cuenta las seis categorías mencionadas antes, al comparar el estatus social del padre con el del esposo. En la tercera medición de movilidad social se tomó en cuenta únicamente los dos estratos principales; ocupación manual y no manual.

19 La diferencia de un año se explica por el hecho de que hay un año de admissao, o sea, cuando el estudiante se prepara para los exámenes que se toman antes de que sea admitido en la escuela secundaria.

20 Las seis categorías de estatus medidas por la ocupación son: 1. Profesionistas y administradores de alto nivel; 2. Gerentes y ejecutivos; 3. Inspectores, supervisores y otras ocupaciones no manuales (nivel alto); 4. Inspectores, supervisores y otras ocupaciones no manuales (nivel bajo); 5. Ocupaciones manuales calificadas; y 6. Ocupaciones manuales semicalificadas y no calificadas. Véase Hutchinson, Bertram, y Carlo Castaldi, "A hierarquia de prestigio das ocupaçoes", en B. Hutchinson (editor), Mobilidade e Trablaho, Río de Janeiro, Centro Brasileiro de Pesquisas Educacionais, 1966, pp. 19-51. 


\section{ANÁLISIS}

Proposición 1. La primera proposición señala que los factores que es$\tan$ relacionados de modo significativo con la ilegitimidad son a) la edad al matrimonio; $b$ ) el credo religioso; c) la religiosidad; $d$ ) el nivel educativo; y e) los antecedentes del estatus social. Los datos que se presentan en el cuadro 1 fundamentan parcialmente esta proposición. Los factores que no discriminan entre las mujeres que tienen o no un hijo natural son: 1) el credo religioso, y 2) la religiosidad.

Sin embargo, debe hacerse notar que a pesar que la edad al matrimonio está significativamente relacionada con la ilegitimidad, tal factor opera en sentido opuesto al predicho. De esta suerte, es más factible que las mujeres que tienen un hijo ilegítimo contraigan motrimonio a mayor edad que aquellas que no han experimentado la ilegitimidad.

\section{Cuadro 1}

Río de Janeiro: Madres Solteras y casadas, SEgún algunas Variables SELECCIONADAS, 1963

\begin{tabular}{|c|c|c|c|}
\hline & $\begin{array}{l}\text { Forciento de } \\
\text { casedas }\end{array}$ & $\begin{array}{l}\text { Porciento de } \\
\text { solteras }\end{array}$ & Total $\mathbf{a}$ \\
\hline$\frac{\text { Edad al matyimonio }}{19: \text { menos }}$ & $\begin{array}{l}95.9 \\
91.8\end{array}$ & $\begin{array}{l}4.1 \\
8.2\end{array}$ & $\begin{array}{l}100.0(677) \\
100.0(947)\end{array}$ \\
\hline$\frac{\text { Religion }}{\text { Católica }}$ & $\begin{array}{l}93.6 \\
92.7\end{array}$ & $\begin{array}{l}6.4 \\
7.3\end{array}$ & $\begin{array}{l}100.0 \\
100.0\left(\begin{array}{l}1389) \\
234\end{array}\right)\end{array}$ \\
\hline $\begin{array}{l}\text { Religiosidad } \mathrm{b} \\
\text { Bo practicante } \\
\text { Semipraoticante } \\
\text { Practicante }\end{array}$ & $\begin{array}{l}94.9 \\
93.1 \\
93.5\end{array}$ & $\begin{array}{l}5.1 \\
6.9 \\
6.5\end{array}$ & 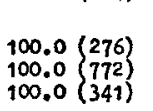 \\
\hline $\begin{array}{l}\frac{\text { Eduoacion }}{\text { linguna }} \\
\text { Elemental } \\
\text { Secundaria o mbs }\end{array}$ & $\begin{array}{l}85.7 \\
92.4 \\
97.6\end{array}$ & $\begin{array}{r}16.7 \\
7.6 \\
2.4\end{array}$ & $\left.\begin{array}{l}100.0(140) \\
100.0 \\
100.0(977) \\
504\end{array}\right)$ \\
\hline $\begin{array}{l}\text { Satatue social del padxe } \\
\text { Alto } \\
\text { Medio } \\
\text { Bajo }\end{array}$ & $\begin{array}{l}99.1 \\
95.4 \\
92.8\end{array}$ & $\begin{array}{l}0.9 \\
4.6 \\
7.2\end{array}$ & $\begin{array}{l}100.0(108) \\
100.0(411) \\
100.0(991)\end{array}$ \\
\hline $\begin{array}{l}\text { Batatua sooial del marido } \\
\text { Alto } \\
\text { Kedio } \\
\text { Bajo }\end{array}$ & $\begin{array}{l}98.0 \\
97.2 \\
90.3\end{array}$ & $\begin{array}{l}2.0 \\
2.8 \\
9.7\end{array}$ & $\begin{array}{l}100.0(205) \\
100.0(492) \\
100.0(877)\end{array}$ \\
\hline$T 0 t a 1$ & 93.5 & 6.5 & $100.0(1626)$ \\
\hline
\end{tabular}

Edad al matrimonio: Ji-cuadrada 10.219, grados de libertad $=1, \mathrm{p}<0.01$; Religión: Ji-cuadrada 0.121 , grados de libertad $=1$, no significativa;

Religiosidad: Ji-cuadrada 1.091, grados de libertad $=2$, no significativa; Educación: Ji-cuadrada 29.718, grados de libertad $=2, \mathrm{p}<0.01$.

Estatus social del padre: Ji-cuadrada 8.657, grados de libertad $=2, \mathrm{p}<0.02$.

Estatus social del marido: Ji-cuadrada 32.482, grados de libertad $=2, \mathrm{p}<0.01$.

a El número total no es igual para cada variable en todos los cuadros debido a la no respuesta.

b Solamente católicas.

Proposición 2. La segunda proposición establece que la variable que se relaciona de manera más significativa con la ilegimitidad es la de los 
antecedentes del estatus social. Los datos del cuadro 1 indican que a pesar de que el porciento de madres solteras se incrementa en forma constante junto con el estatus social del padre, esta medición de los antecedentes sociales no es estadísticamente el factor más importante relacionado con la ilegitimidad. El nivel educativo de la mujer y el estatus social de su marido parecen ser estadísticamente más significativos.

Los datos presentados en el cuadro 2 indican la relevancia que tiene el estatus social del padre en comparación con las otras variables. Se hicieron pruebas de significancia para cada variable dentro de cada uno de los dos niveles del estatus (tal y como lo mide el estatus social del padre). Una vez que los antecedentes del estatus social sean controlados, el nivel educativo y el estatus social del esposo son los factores que continúan siendo significativamente relacionados con la ilegitimidad. La edad al matrimonio continúa siendo significativa únicamente en el nivel de ocupación manual, mientras que en el nivel no manual, este factor deja de discriminar entre madres solteras y casadas. Más aún, en el nivel de las ocupaciones manuales, los resultados se presentan en sentido opuesto del que se predijo, de tal modo que es más verosímil que las mujeres que tienen hijos naturales sean mayores que las mujeres que no los tienen al momento de casarse.

Tanto el credo religioso como la religiosidad mantienen su no significación respecto a la ilegitimidad.

En resumen, el análisis que se presenta en los cuadros 1 y 2 indica que las cuantificaciones sobre la religión no están relacionadas de manera significativa con el fenómeno de la ilegitimidad. La edad al matrimonio, el nivel de educación, el estatus social del padre y el estatus social del marido sí están significativamente relacionados con el hecho de tener hijos naturales. Cuando se controla el factor de los antecedentes del estatus social (medido a través del estatus social del padre), se mantiene una relación significativa entre edad al matrimonio, nivel educativo y estatus social del esposo con la ilegitimidad. Además, los datos de los cuadros 1 y 2 indican que los antecedentes de clase social no constituyen el factor más importante en relación con la ilegitimidad. El nivel educativo de la mujer y el estatus social del marido resultan estadísticamente más significativos.

Puede discutirse que el estatus social del padre es ciertamente el factor de mayor significación entre los antecedentes, y que el nivel educativo y el estatus social del marido son consecuencias de la ilegitimidad muy significativas. Si este argumento fuera válido, entonces se podría sostener que el hecho de tener un hijo natural acarrea importantes consecuencias para la movilidad social de la madre, medidas a través del nivel educativo alcanzado $\mathrm{y}$ en forma particular por el estatus social del marido.

Proposición 3. La tercera proposición establece que es más probable que las mujeres socialmente estables tengan hijos ilegítimos que aquellas que observan un progreso ascendente, y menos probable que aquellas que experimentan un descenso.

El cuadro 3 ofrece datos sobre movilidad, sentido de la movilidad y movilidad a través de estratos sociales. Si se considera sólo la condición de movilidad, parece que es menos probable que lleguen a la ilegitimidad las mujeres con movilidad social que aquellas que permanecen en 
Cuadro 2

Río de Janeiro: Estatus social de madres solteras y Casadas, por variables SELECCIONADAS, 1963

\begin{tabular}{|c|c|c|c|c|c|c|c|c|}
\hline & \multicolumn{8}{|c|}{ Es tatue Booisl del padre } \\
\hline & $\begin{array}{l}\text { Porolento } \\
\text { de oseadas }\end{array}$ & $\begin{array}{l}\text { loion no manus } \\
\text { Porionto to } \\
\text { de soltexes }\end{array}$ & Toto & & $\begin{array}{l}\text { Poralento } \\
\text { de ossadss }\end{array}$ & $\begin{array}{l}\text { acion mamual } \\
\text { Poroiento } \\
\text { do solteras }\end{array}$ & Tote & \\
\hline $\begin{array}{l}\text { Idsd al matrimonio } \\
19 \text { años or monaso } \\
20 \text { años o mAs }\end{array}$ & $\begin{array}{l}95.8 \\
96.3\end{array}$ & $\begin{array}{l}4.2 \\
3.7\end{array}$ & $\begin{array}{l}100.0 \\
100.0\end{array}$ & $\left\{\begin{array}{l}167 \\
354\end{array}\right)$ & $\begin{array}{l}96.1 \\
88.4\end{array}$ & $\begin{array}{r}3.9 \\
11.6\end{array}$ & $\begin{array}{l}100.0 \\
100.0\end{array}$ & $\left\{\begin{array}{c}459 \\
541\end{array}\right\}$ \\
\hline 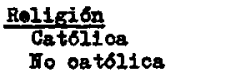 & $\begin{array}{l}96.2 \\
96.0\end{array}$ & $\begin{array}{l}3.8 \\
4.0\end{array}$ & $\begin{array}{l}100.0 \\
100.0\end{array}$ & $\left\{\begin{array}{l}442) \\
75)\end{array}\right.$ & $\begin{array}{l}92.1 \\
90.8\end{array}$ & $\begin{array}{l}7.9 \\
9.2\end{array}$ & $\begin{array}{l}100.0 \\
100.0\end{array}$ & $\left\{\begin{array}{c}858 \\
142\end{array}\right)$ \\
\hline $\begin{array}{l}\text { Religiosidad } \\
\text { Fo practioante } \\
\text { Samipractioante } \\
\text { Praotioante }\end{array}$ & $\begin{array}{l}94.3 \\
96.3 \\
97.1\end{array}$ & $\begin{array}{l}5.7 \\
3.7 \\
2.9\end{array}$ & $\begin{array}{l}100.0 \\
100.0 \\
100.0\end{array}$ & $\left\{\begin{array}{l}88) \\
2166 \\
138\end{array}\right)$ & $\begin{array}{l}94.3 \\
91.8 \\
91.1\end{array}$ & $\begin{array}{l}5.7 \\
8.2 \\
8.9\end{array}$ & $\begin{array}{l}100.0 \\
100.0 \\
100.0\end{array}$ & $\left\{\begin{array}{l}158 \\
510 \\
190\end{array}\right)$ \\
\hline 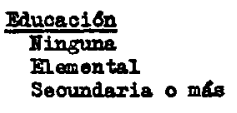 & $\begin{array}{l}100.0 \\
92.4 \\
98.2\end{array}$ & $\begin{array}{l}0.0 \\
7.6 \\
1.8\end{array}$ & $\begin{array}{l}100.0 \\
100.0 \\
100.0\end{array}$ & $\left\{\begin{array}{l}7) \\
844 \\
325\end{array}\right)$ & $\begin{array}{l}84.7 \\
92.1 \\
96.9\end{array}$ & $\begin{array}{r}15.3 \\
7.9 \\
3.1\end{array}$ & $\begin{array}{l}100.0 \\
100.0 \\
100.0\end{array}$ & 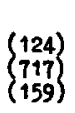 \\
\hline Estatus sooisl dol & & & & & & & & \\
\hline $\begin{array}{c}\frac{\text { esposo }}{\text { M1to }} \\
\text { Modio } \\
\text { Bajo }\end{array}$ & $\begin{array}{l}98.2 \\
96.9 \\
91.7\end{array}$ & $\begin{array}{l}1.8 \\
3.1 \\
8.3\end{array}$ & $\begin{array}{l}100.0 \\
100.0 \\
100.0\end{array}$ & $\left\{\begin{array}{l}164 \\
226 \\
121\end{array}\right)$ & $\begin{array}{l}97.2 \\
97.1 \\
89.4\end{array}$ & $\begin{array}{r}2.8 \\
2.9 \\
11.6\end{array}$ & $\begin{array}{l}100.0 \\
100.0 \\
100.0\end{array}$ & $\left(\begin{array}{l}36) \\
239 \\
683\end{array}\right)$ \\
\hline
\end{tabular}

Ocupación no manual: Edad al matrimonio: Ji-cuadrada 0.002 , grados de libertad $=1$, no significativa; Religión: Ji-cuadrada 0.067 , grados de libertad $=1$, no significativa; Religiosidad: Ji-cuadrada 1.149, grados de libertad $=2$, no significativa; Educación: Ji-cuadrada 9.724, grados de libertad $=1, \mathrm{p}<0.01$; Estatus social del esposo: Ji-cuadrada 8.131, grados de libertad $=1, \mathrm{p}<0.01$

Ocupación manual: Edad al matrimonio: Ji-cuadrada 18.875, grados de libertad $=1, \mathrm{p}<0.01$; Religión: Jicuadrada 0.110 , grados de libertad $=1$, no significativa; Religiosidad: Ji-cuadrada 1.415 , grados de libertad $=$ 1, no significativa; Educación: Ji-cuadrada 13.956, grados de libertad $=1,0.01>\mathrm{p}>0.02$; Estatus social del esposo: Ji-cuadrada 14.558, grados de libertad $=1, \mathrm{p}<0.01$ 
una misma condición. Sin embargo, la diferencia no es estadísticamente significativa. Cuando se toma en cuenta el sentido de la movilidad, otra vez parece que los datos confirman las predicciones cuando menos respecto a la movilidad ascendente. No obstante, las pruebas estadísticas no resultaron significativas. Cuando los antecedentes del estatus social se mantienen constantes, entonces los resultados sí son estadísticamente significativos. De esta manera, al mismo tiempo que los antecedentes sociales son un importante factor en la explicación de la ilegitimidad, también la movilidad social resulta un factor explicativo significativo para comprender este fenómeno.

\section{Cuadro 3}

Río de JANEIro: Movilidad SOCIAL de MADRES CASADAS Y SOLTERAS, POR SENTIDO DE LA MOVILIDAD Y ESTATUS SOCIAL, 1963

\begin{tabular}{|c|c|c|c|}
\hline & $\begin{array}{l}\text { Porciento do } \\
\text { oagadas }\end{array}$ & $\begin{array}{l}\text { Porciento de } \\
\text { solteras }\end{array}$ & Total \\
\hline $\begin{array}{c}\text { Movilidad social } \\
\text { Con movilidad } \\
\text { Sin movilidad }\end{array}$ & $\begin{array}{l}93.9 \\
92.2\end{array}$ & $\begin{array}{l}6.1 \\
7.8\end{array}$ & $\begin{array}{l}100.0(922) \\
100.0(548)\end{array}$ \\
\hline $\begin{array}{c}\text { Sontido de le movilidad } \\
\text { Kovilidad ascenderte } \\
\text { Sin movilidad } \\
\text { Xovilidad desoendento }\end{array}$ & $\begin{array}{l}94.3 \\
92.2 \\
93.0\end{array}$ & $\begin{array}{l}5.7 \\
7.8 \\
7.0\end{array}$ & $\begin{array}{l}100.0 \\
100.0 \\
100.0\end{array}\left\{\begin{array}{l}652 \\
548 \\
270\end{array}\right)$ \\
\hline $\begin{array}{c}\text { Hovilidad a traver de egtrat } \\
\text { Oeupsciones no manuale } \\
\text { Sin movilidad } \\
\text { Desoendente b }\end{array}$ & $\begin{array}{l}97.4 \\
91.7\end{array}$ & $\begin{array}{l}2.6 \\
8.3\end{array}$ & $\begin{array}{l}100.0 \\
100.0\left(\begin{array}{l}390 \\
121\end{array}\right)\end{array}$ \\
\hline $\begin{array}{l}\text { Ocupaciones manuales } \\
\text { Ascendente b/ } \\
\text { Sin movilidad }\end{array}$ & $\begin{array}{l}97.1 \\
89.6\end{array}$ & $\begin{array}{r}2.9 \\
10.4\end{array}$ & $\begin{array}{l}100.0 \\
100.0\end{array}\left\{\begin{array}{l}275 \\
684\end{array}\right\}$ \\
\hline
\end{tabular}

Movilidad social: Ji-cuadrada 1.449 , grados de libertad $=1$, no significativa.

Sentido de la movilidad: Ji-cuadrada 2.284 , grados de libertad $=2$, no significativa. Movilidad a travás de estratos: Ocupación no manual: Ji-cuadrada 6.538 , grados de libertad $=1, \mathrm{p}<0.01$.

Movilidad a través de estratos: Ocupación manual: Ji-cuadrada 13.512, grados de libertad $=1, \mathrm{p}<0.01$.

a Movilidad a través de seis categorías sociales.

b Cruce de la separación entre estratos de ocupación manual y no manual.

Proposición 4. La cuarta proposición establece que los factores que afectan el lapso transcurrido entre el nacimiento de un hijo natural y el matrimonio subsecuente, son: a) la edad al matrimonio; b) el credo religioso; c) la religiosidad; d) el nivel educativo, y $e$ ) los antecedentes del estatus social.

Los datos del cuadro 4 no apoyan esta proposición. Aunque algunos de los factores llevan el sentido esperado (véanse las medianas del cuadro 4), ninguna de las pruebas estadísticas resulta significativa. El factor que más se aproxima al nivel de significancia es el estatus del esposo.

Las cifras del cuadro 5 indican la relación entre algunas variables seleccionadas cuando se aíslan los antecedentes del estatus social. La mayoría de las pruebas de significancia dentro del antecedente del estatus social no apoyan esa cuarta proposición. La única prueba que llena el 


\section{Cuadro 4}

Río de JANEIRo: LAPSO ENTRE LA MATERNIDAd SIN MATRIMONIO Y EL CASAMIENTO SUBSECUENTE, POR VARIABLES SELECCIONADAS, 1963

\begin{tabular}{|c|c|c|c|c|}
\hline & $\begin{array}{l}\text { Un año } 0 \\
\text { menos }\end{array}$ & $\begin{array}{l}\text { Más de } \\
\text { un año }\end{array}$ & Total & $\begin{array}{c}\text { Mediana del intervalo } \\
\text { (on meses) }\end{array}$ \\
\hline 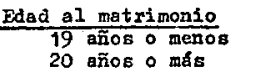 & $\begin{array}{l}53.6 \\
42.3\end{array}$ & $\begin{array}{l}46.4 \\
57.7\end{array}$ & $\begin{array}{l}100.0 \\
190.0\end{array}\left(\begin{array}{l}28 \\
78\end{array}\right)$ & $\begin{array}{l}10.0 \\
19.0\end{array}$ \\
\hline 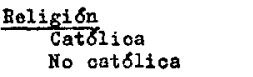 & $\begin{array}{l}43.8 \\
52.9\end{array}$ & $\begin{array}{l}56.2 \\
47.1\end{array}$ & $\left.\begin{array}{l}100.0(89 \\
100.0 \\
17\end{array}\right)$ & $\begin{array}{r}17.0 \\
8.0\end{array}$ \\
\hline $\begin{array}{l}\frac{\text { Beligiosidad }}{\text { No practicante }} \\
\text { Semiprecticanto } \\
\text { Practicante }\end{array}$ & $\begin{array}{l}28.6 \\
47.2 \\
45.5\end{array}$ & $\begin{array}{l}71.4 \\
52.8 \\
54.5\end{array}$ & $\begin{array}{l}100.0\left(\begin{array}{l}14 \\
100.0 \\
53 \\
100.0(22\end{array}\right)\end{array}$ & $\begin{array}{l}16.5 \\
22.0 \\
14.0\end{array}$ \\
\hline $\begin{array}{l}\frac{\text { Educacion }}{\text { Ninguna }} \\
\text { Elemental } \\
\text { Securdario o mas }\end{array}$ & $\begin{array}{l}35.0 \\
45.9 \\
58.3\end{array}$ & $\begin{array}{l}65.0 \\
54.1 \\
41.7\end{array}$ & $\begin{array}{l}100.0(20) \\
900.0(74) \\
100.0(12)\end{array}$ & $\begin{array}{l}19.0 \\
16.0 \\
10.0\end{array}$ \\
\hline 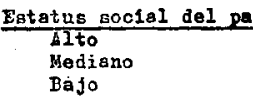 & $\begin{array}{r}100.0 \\
42.1 \\
46.9\end{array}$ & $\begin{array}{r}0.0 \\
57.9 \\
53.1\end{array}$ & $\begin{array}{l}100.0\left(\begin{array}{l}1) \\
100.0 \\
100.0\end{array}\left(\begin{array}{l}19 \\
81\end{array}\right)\right.\end{array}$ & $\begin{array}{l}(-) \\
13.5 \\
17.0\end{array}$ \\
\hline $\begin{array}{l}\text { Egtatus social ael os } \\
\text { Alto } \\
\text { Mediano } \\
\text { Bajo }\end{array}$ & $\begin{array}{l}75.0 \\
57.1 \\
41.2\end{array}$ & $\begin{array}{l}25.0 \\
42.9 \\
58.8\end{array}$ & $\begin{array}{l}100.0(4) \\
100.0(14) \\
100.0(85)\end{array}$ & $\begin{array}{r}7.5 \\
8.0 \\
17.0\end{array}$ \\
\hline T 0 : 1 & 45.3 & 54.7 & $900.0(106)$ & 15.0 \\
\hline
\end{tabular}

Edad al matrimonio: Ji-cuadrada 0.649 , grados de libertad $=1$, no significativa; Religión: Ji-cuadrada 0.185 , grados de libertad $=1$, no significativa;

Religiosidad: Ji-cuadrada 1.588, grados de libertad $=2$, no significativa;

Educación: Ji-cuadrada 1.691, grados de libertad $=2$, no significativa;

Estatus social del padre: Ji-cuadrada 0.022 , grados de libertad $=1$, no significativa;

Estatus social del esposo: Ji-cuadrada 2.452 , grados de libertad $=1$, no significativa.

requisito de ser significativa es el estatus del esposo en aquellas mujeres con antecedentes de padres con ocupación no manual.

Así, aun cuando estas cifras son muy sugestivas, fallan en confirmar la cuarta proposición.

Proposición 5. Esta quinta proposición sugiere que el factor relacionado de manera más significativa con el intervalo transcurrido entre la ilegitimidad y el matrimonio subsecuente es el de la clase social como antecedente.

Como se observa en el cuadro 4, la conexión entre el estatus del padre y el intervalo resulta menor que entre otros factores y este lapso. Además, los datos del cuadro 5 no siempre indican un mayor efecto del estatus del padre que de los otros factores. Así, esta proposición no se encuentra apoyada por los datos.

De hecho, y como se hizo notar anteriormente, el estatus social del marido es el único factor que muestra una conexión con el tiempo, que se aproxima más a la significación, y que, cuando se mantiene fijo el estatus del padre, se muestra significativo, aunque sólo sea entre aquellas 


\section{Cuadro 5}

Río DE JANEIRO: LAPSO ENTRE MATERNIDAD SIN MATRIMONIO Y EL CASAMIENTU SUBSECUENTE POR ESTATUS SOCIAL Y ALGUNAS VARIABLES SELECCIONADAS, 1963

\begin{tabular}{|c|c|c|c|c|c|c|}
\hline & \multicolumn{6}{|c|}{ Estatus oooiel del padre } \\
\hline & \multicolumn{3}{|c|}{ Ooupaojón no manual } & \multicolumn{3}{|c|}{ Ooupaoion manual } \\
\hline & $\begin{array}{l}\text { Un año } \\
\text { menos }\end{array}$ & $\begin{array}{l}\text { Mog do } \\
\text { un año }\end{array}$ & Total & $\begin{array}{l}\text { Un año } 0 \\
\text { menos }\end{array}$ & $\begin{array}{l}\text { UK! de } \\
\text { un año }\end{array}$ & Total 1 \\
\hline \multicolumn{7}{|l|}{ Edad al atrimonio } \\
\hline $\begin{array}{l}19 \text { añios o menos } \\
20 \text { años o nós }\end{array}$ & $\begin{array}{l}42.9 \\
46.2\end{array}$ & $\begin{array}{l}57.1 \\
53.8\end{array}$ & $\begin{array}{l}100.0(7) \\
100.0\end{array}$ & $\begin{array}{l}61.9 \\
42.9\end{array}$ & $\begin{array}{l}38.9 \\
57.1\end{array}$ & $\begin{array}{l}100.0 \\
100.0\end{array}\left\{\begin{array}{l}18 \\
63\end{array}\right\}$ \\
\hline \multicolumn{7}{|l|}{ Beligion } \\
\hline $\begin{array}{l}\text { Catolica } \\
\text { Io oatolioa }\end{array}$ & $\begin{array}{l}41.2 \\
66.7\end{array}$ & $\begin{array}{l}58.8 \\
33.3\end{array}$ & $\begin{array}{l}100.0\left(\begin{array}{l}17) \\
100.0 \\
3\end{array}\right)\end{array}$ & $\begin{array}{l}47.1 \\
46.2\end{array}$ & $\begin{array}{l}52.9 \\
53.8\end{array}$ & $\begin{array}{l}100.0 \\
100.0\end{array}\left(\begin{array}{l}68 \\
13\end{array}\right)$ \\
\hline \multicolumn{7}{|l|}{ 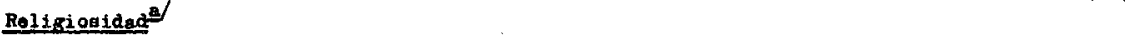 } \\
\hline $\begin{array}{l}\text { No prootioante } \\
\text { Semipractioante } \\
\text { Prootioante }\end{array}$ & $\begin{array}{l}40.0 \\
25.0 \\
75.0\end{array}$ & $\begin{array}{l}60.0 \\
75.0 \\
25.0\end{array}$ & $\begin{array}{l}100.0 \\
100.0 \\
100.0\end{array}\left\{\begin{array}{l}5 \\
4\end{array}\right\}$ & $\begin{array}{l}22.2 \\
54.8 \\
41.2\end{array}$ & $\begin{array}{l}77.8 \\
45.2 \\
58.8\end{array}$ & $\begin{array}{l}100.0 \\
100.0 \\
100.0\end{array}\left\{\begin{array}{l}9 \\
42 \\
17\end{array}\right\}$ \\
\hline \multicolumn{7}{|l|}{ Edugagion } \\
\hline $\begin{array}{l}\text { Kinguna } \\
\text { Biemontal } \\
\text { Seoundaria o mas }\end{array}$ & $\begin{array}{r}0.0 \\
42.9 \\
50.0\end{array}$ & $\begin{array}{r}0.0 \\
57.1 \\
50.0\end{array}$ & $\begin{array}{l}100.0(0) \\
100.0\left(\begin{array}{l}14) \\
100.0\end{array}(6)\right.\end{array}$ & $\begin{array}{l}36.8 \\
47.4 \\
80.0\end{array}$ & $\begin{array}{l}63.2 \\
52.6 \\
20.0\end{array}$ & $\begin{array}{l}100.0 \\
100.0 \\
100.0\end{array}\left(\begin{array}{l}19 \\
57 \\
5\end{array}\right)$ \\
\hline \multicolumn{7}{|c|}{ Fetatup sooisl del eaposo } \\
\hline $\begin{array}{l}\text { Mlto } \\
\text { Madio } \\
\text { Bajo }\end{array}$ & $\begin{array}{r}100.0 \\
57.1 \\
20.0\end{array}$ & $\begin{array}{r}0.0 \\
42.9 \\
80.0\end{array}$ & $\begin{array}{l}100.0(3) \\
100.0\{7 \\
100.0(10)\end{array}$ & $\begin{array}{r}0.0 \\
57.1 \\
45.1\end{array}$ & $\begin{array}{r}100.0 \\
42.9 \\
54.9\end{array}$ & $\begin{array}{l}100.0\{1 \\
100.0\{7 \\
100.0(71)\end{array}$ \\
\hline
\end{tabular}

Ocupación no manual: Edad al matrimonio: Ji-cuadrada 0.109, grados de libertad $=1$, no significativa; Religión: Ji-cuadrada 0.036 , grados de libertad $=1$, no significativa (NR): Religiosidad: Ji-cuadrada 2.756, grados de libertad $=2$, no significativa; Educación: Ji-cuadrada 0.087 , grados de libertad $=2$, no significativa (NR); Estatus social de esposo: Ji-cuadrada 5.018, grados de liber$\operatorname{tad}=1,0.02<\mathrm{p}<0.05$.

Ocupación manual: Edad al matrimonio: Ji-cuadrada 1.219, grados de libertad $=$ 1 , no significativa; Religión: Ji-cuadrada 0.057 , grados de libertad $=1$, no significativa; Religiosidad: Ji-cuadrada 0.314 , grados de libertad $=1$, no significativa; Educación: Ji-cuadrada 1.042, grados de libertad $=1$, no significativa; Estatus social del esposo: Ji-cuadrada 1.222, grados de libertad $=2$, no significativa.

a Sólo católicos.

mujeres de padres con ocupaciones no manuales. Esta relación entre el intervalo y el estatus del marido es sólo sugerente, pero pudiera señalar consecuencias que la ilegitimidad acarrea para la movilidad social de las madres solteras.

Proposición 6. La sexta proposición establece que a las madres solteras que son estables socialmente, les lleva más tiempo casarse que a aquellas que progresan socialmente, aunque menos que las que descienden en la escala social. El cuadro 6 presenta información relativa a esta proposición.

La movilidad en sí no discrimina entre aquellas que se casan pronto y aquellas que esperan más para casarse. Cuando se toma en cuenta el sentido de la movilidad, la información parece avalar las hipótesis hechas, 


\section{Cuadro 6}

RÍO DE JANEIRO: LAPSO TRANSCURRIDO ENTRE LA MATERNIDAD SIN MATRIMONIO Y EL SUBSECUENTE CASAMIENTO, POR MOVILIDAD SOCIAL, SENTIDO DE LA MOVILIDAD, Y ESTATUS SOCIAL, 1963

\begin{tabular}{|c|c|c|c|c|}
\hline & $\begin{array}{l}\text { Un año } \circ \\
\text { menos }\end{array}$ & $\begin{array}{l}\text { Más de } \\
\text { un año }\end{array}$ & Total & $\begin{array}{l}\text { Mediana del inter- } \\
\text { valo (en mesec) }\end{array}$ \\
\hline $\begin{array}{l}\text { nbvil } \\
\text { Inmovil }\end{array}$ & $\begin{array}{l}46.2 \\
44.2\end{array}$ & $\begin{array}{l}53.6 \\
55.8\end{array}$ & $\begin{array}{l}100.0 \\
100.0\end{array} \quad\left(\begin{array}{l}56 \\
43\end{array}\right)$ & $\begin{array}{r}7.0 \\
19.0\end{array}$ \\
\hline $\begin{array}{l}\text { Sentido de la movilided } \\
\text { Kovilidad a scendente } \\
\text { Inm6ril } \\
\text { Movilidad desoendente }\end{array}$ & $\begin{array}{l}54.1 \\
44.2 \\
31.6\end{array}$ & $\begin{array}{l}45.9 \\
55.8 \\
68.4\end{array}$ & $\begin{array}{ll}100.0 & (37) \\
100.0 & (43) \\
100.0 & (19)\end{array}$ & $\begin{array}{r}8.0 \\
19.0 \\
25.0\end{array}$ \\
\hline 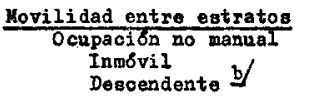 & $\begin{array}{l}70.0 \\
20.0\end{array}$ & $\begin{array}{l}30.0 \\
80.0\end{array}$ & $\begin{array}{l}100.0 \\
100.0\end{array} \quad\left(\begin{array}{l}10 \\
10\end{array}\right)$ & $\begin{array}{r}6.0 \\
20.0\end{array}$ \\
\hline $\begin{array}{l}\text { Ooupecion manus } \\
\text { Asoendente b/ } \\
\text { Inmovil }\end{array}$ & $\begin{array}{l}50.0 \\
45.1\end{array}$ & $\begin{array}{l}50.0 \\
54.9\end{array}$ & $\begin{array}{l}100.0 \\
100.0 \quad(8) \\
71)\end{array}$ & $\begin{array}{l}17.5 \\
15.0\end{array}$ \\
\hline
\end{tabular}

Movilidad social: Ji-cuadrada 0.000 , grados de libertad $=1$, no significativa;

Sentido de la movilidad: Ji-cuadrada 2.607, grados de libertad $=2$, no significativa; Movilidad entre estratos: Ocupación no manual: Ji-cuadrada 3.232, grados de libertad $=1,0.05>\mathrm{p}>0.10$; Ocupación manual: Ji-cuadrada 0.011, grados de libertad $=1$, no significativa;

a Movilidad a través de las seis categorías de estatus.

b Atravesando la separación de los estratos de ocupación manual y no manual.

aunque las pruebas estadísticas no alcanzan a ser significativas. Cuando se toman en cuenta tanto el sentido de la movilidad, como el cruce de la separación del estatus, los datos parecen confirmar nuevamente el sentido indicado, pero no son estadísticamente significativos. La única comparación que se aproxima bastante a la significancia es la de la movilidad e inmovilidad social de las mujeres de padres con ocupación no manual.

En resumen, a pesar de que las medianas del cuadro 6 son altamente sugerentes, los datos no confirman esta proposición.

\section{Comentarios}

Los factores más sobresalientes en relación con la ilegitimidad son tanto el estatus social del padre como el del esposo, el primero como una variable de antecedente, y el segundo como una consecuencia. Tales hechos son consistentes con la historia social del Brasil. Freyre ${ }^{21}$ en su trabajo clásico Casa grande e senzala describe la estructura de la familia tradicional de ese país. La familia como institución existió en los niveles altos de la sociedad, mientras que los esclavos no constituían familias como lo hacían los señores. Las esclavas y las mujeres de clase 1958.

21 Gilberto Freyre, Casa Grande e Zenzala, Río de Janeiro, José Olimpio, 
baja solian tener hijos de padres de esas mismas categorías, además de que en muchas ocasiones tenían hijos con los amos. Tal y como se definió, la ilegitimidad parece haber sido la forma común de reproducción en los bajos niveles de la sociedad. A pesar de que se haya abolido la esclavitud, aún se puede encontrar tal patrón de conducta en algunas áreas rurales del Brasil. Muchas familias se constituyen a través del mutuo consentimiento y este fenómeno no es fuera de lo común en muchas regiones del país. También se da en sectores de grandes ciudades donde hay una concentración de gente de clase baja de origen rural. ${ }^{22}$

$\mathrm{Si}$ se supone que la familia como institución es una función de la clase social, se esperaría que las tasas de ilegitimidad fueran mayores en los estratos más bajos de la sociedad, tal y como se demostró anteriormente. Dado que la educación es también función de la clase social, los hechos encontrados que se presentan son consistentes con aquellos que se muestran en la literatura sobre estratificación social. Empero, la religión no guarda el sentido esperado. En Brasil tanto la religión como la religiosidad parecen ser indicadores débiles del comportamiento futuro. Aunque la práctica del catolicismo se relaciona ligeramente con la clase social, ${ }^{23}$ parece que la socialización religiosa no es tan eficiente como para evitar que se tengan hijos ilegítimos en contra de las enseñanzas de la iglesia. En otras palabras, la religiosidad - como asistencia a la iglesia - parece no ser suficiente para contraatacar los valores subculturales y los patrones de conducta. Sin embargo, es posible que la religión actúe en dos sentidos. Si existe una asociación directa entre ser católico de nombre y pertenecer a la clase baja, se esperaría una mayor proporción de mujeres que han dado lugar a la ilegitimidad entre las católicas no practicantes que entre las semipracticantes, y una menor proporción entre las católicas practicantes. Aunque pueden encontrarse diferencias entre los dos extremos, la asociación no se mantiene cuando se consideran todas las católicas, y sus diferencias no son significativas. Cuando se aislan los antecedentes del estatus social, la dirección de la ilegitimidad entre las ocupaciones no manuales es inversa de aquella entre las ocupaciones manuales. Es posible que lo encontrado pueda explicarse por el tipo de mediciones adoptadas, y que la asistencia a la iglesia pueda ser una estimación inadecuada de la religiosidad, pudiendo ser que la asistencia a la iglesia refleje únicamente un tipo de actividad social en Brasil. Si se supone que no es sólo una actividad social, podemos intentar una explicación alternativa. Puede suceder que las católicas no practicantes de clase baja se comporten de acuerdo con su patrón subcultural. Por otra parte, es posible que las católicas practicantes de clase baja se comporten de acuerdo con el patrón impuesto por la clase social, y que la religión refuerce la ilegitimidad porque la iglesia católica condena el aborto. En otras palabras, cuando una mujer se embaraza puede ser que su comportamiento se deba a que las relaciones sexuales antes del matrimonio sean relativamente aceptadas en el estrato en que ella vive, y el

22 Sagmacs, Aspectos Humanos da Favela Carioca, O Estados de São Paulo, São Paulo, 13-15 de abril de 1960, parte 2, pp. 3-6.

23 El "estatus social medio" de los católicos no practicantes es 4.42; de los semipracticantes 4.49; y de los practicantes 4.00. La media para los católicos es $4.35 \mathrm{y}$ de 4.14 para los no católicos. La media se calculó utilizando las seis categorías de estatus mencionadas antes, tomándose la categoría 1 como la más alta. 
hecho de que también sea católica practicante podría funcionar como un factor acumulativo que la lleve a la ilegitimidad.

Un hallazgo interesante es que la edad al matrimonio está significativamente relacionada con la ilegitimidad aunque los datos son contrarios a lo esperado. Es posible que esta relación demuestre la "protección" de la familia. Esto es, mientras más joven es la mujer, es más factible que sea presionada por sus padres o parientes para que se case y entonces tenga hijos. También es posible que mientras más joven sea la mujer, mayor es la posibilidad de que sea aceptada como esposa. Más aún, si se embaraza cuando ya es mayor la hace más responsable de sus actos, y a su vez disminuye la oportunidad de que se case.

Cuando se comparaba el estatus social del padre con el del marido, se encontró que este último es un factor más significativo. Puesto que ambos factores reflejan el estatus social de la mujer en distintos períodos de su vida, la información muestra que la ilegitimidad está asociada con la movilidad social. Es menos probable que una mujer socialmente ascendente tenga un hijo natural que aquella que conserva o declina su nivel social. Puede apreciarse este panorama general cuando se aisla la movilidad social por los estratos sociales. Como se mencionó antes, las mujeres de menor nivel social llegan a la ilegitimidad con más frecuencia que las mujeres del nivel superior. En otras palabras, mientras que una mujer en el estrato de ocupación no manual llega a la ilegitimidad, en el nivel de ocupación manual cuatro hacen lo mismo. Por otra parte, es menos probable que tengan hijos ilegítimos aquellas mujeres que socialmente ascienden, cruzando la separación entre las ocupaciones no manuales y manuales, que otras de su mismo estrato de origen. Lo inverso también es verdadero. Las mujeres que socialmente descienden están más expuestas a tener hijos ilegítimos que aquellas que permanecen estables socialmente. Se puede concluir entonces que la gente con movilidad social se comporta de manera distinta que aquella que siendo originaria del mismo estrato permanece en él, pero aún no pueden compararse con la gente que pertenece al estrato hacia el cual se mueven. ${ }^{24}$

$\mathrm{Si}$ consideramos ahora el tiempo que transcurre para que una mujer con un hijo ilegítimo se case, aunque sólo una de las variables discrimine significativamente, la dirección que tomó el matrimonio subsecuente es consistente con el hecho de la ilegitimidad. Por tal razón se añade cierta dificultad. El tiempo que tarda una mujer con hijos ilegítimos en casarse parece estar asociado con su educación y el estatus social del padre. Esto es, mientras mayor es la educación y el estatus social de las mujeres, requieren menor tiempo para formar una familia. Otra vez, la religión y la religiosidad no son factores importantes. Más bien la fecha del matrimonio después del nacimiento del hijo parece ser más una función del estatus social. Los datos sugieren que aquellas mujeres de altos niveles están más conscientes del problema que es tener un hijo natural y por lo tanto ponen más empeño para remediar esa situación que el que ejercen las mujeres de estratos bajos. Lo mismo parece ser válido para las mujeres

24 Cf. Bertram Hutchinson, "Colour, Social Status and Fertility in Brazil", America Latina, Vol. 8, Núm. 4, octubre-diciembre de 1965, p. 22, donde el autor hace notar que las mujeres con movilidad social muestran un esquema de fertilidad que cae entre el del nivel de estatus que dejan y el del nivel al que se incorporan. 
jóvenes; para éstas posiblemente sea más factible contraer matrimonio antes que para las mayores porque sus padres pueden ejercer cierto tipo de presión que las conduzca a un pronto matrimonio. Sin embargo, si este patrón es válido para el total de la población, parece haber una diferencia cuando se toman como constantes los orígenes sociales de las mujeres. Los datos del cuadro 5, parecen confirmar el esquema para el nivel de ocupación manual, pero no para el de ocupación no manual. Es posible que la distribución encontrada en este último grupo se deba al tamaño de la muestra.

Tal como se observó antes, la movilidad social puede explicar la variación encontrada en la probabilidad de tener hijos naturales, pero el panorama no es claro respecto al tiempo que toma a las mujeres para que contraigan nupcias. En general, los datos demuestran (véase el cuadro 6), que para aquellas que ascienden socialmente, es más factible que les lleve menos tiempo casarse que las que son estables y que las que descienden, pero a nada concluyente puede llegarse. La única excepción puede encontrarse entre las mujeres que guardan estabilidad y las que descienden dentro del nivel de ocupación no manual. Si se supone que los resultados encontrados muestran al menos cierta dirección, es posible decir que para aquellas que al casarse descienden socialmente, es más probable que les tome más tiempo establecer un matrimonio que para aquellas que permanecen en el mismo estrato social.

\section{CONCLUSIONES}

Esta investigación demuestra que los antecedentes del estatus social de una mujer predicen bien la ilegitimidad. Sin embargo, el mejor factor aislado es el estatus social del marido. Cuando se comparan ambos estatus sociales (esto es, movilidad social), los datos indican que cruzar la separación entre ocupaciones de tipo no manual y manual se relaciona significativamente con la ilegitimidad. Las mujeres con antecedentes de ocupaciones no manuales y que descienden socialmente, tienden a tener más hijos naturales que aquellas que permanecen en ese nivel social. Por otra parte, las mujeres con antecedentes de ocupaciones manuales y que ascienden socialmente hacia el estrato de ocupación no manual, están menos expuestas a llegar a la ilegitimidad que aquellas que permanecen en ese nivel. También se encontró que la educación se relaciona con la ilegitimidad, y que las mujeres menos educadas están más predispuestas a tener un hijo ilegítimo. Ni el credo religioso, ni la religiosidad, logran hacer distinciones. Se encontró que la edad al matrimonio se asocia con la ilegitimidad en el sentido opuesto al predicho, esto es, es más probable que hayan tenido hijos ilegítimos aquellas mujeres que se casan a mayor edad.

Todos los fenómenos encontrados respecto a la ilegitimidad, son consistentes con aquellos respecto al lapso que existe entre el nacimiento del primer hijo y el matrimonio subsecuente. Esto es, para las mujeres que pertenecen a un estrato social elevado y que han alcanzado un nivel educativo alto es más probable que se casen en un periodo más corto. Más aún, es más probable que les tome menos tiempo a aquellas que sa casan jóvenes. Repitiendo, la religión y la religiosidad no logran discri- 
minar. Los datos dan a entender que las mujeres con ascendencia social tienden a requerir menos tiempo en casarse que aquellas que son estables o que descienden socialmente.

Los resultados de la investigación indican que en Brasil la familia como institución es una función del estatus social, tal y como lo han señalado los historiadores sociales. En otras palabras, los componentes de las clases bajas todavía presentan, hasta cierto grado, rasgos observados en el pasado por miembros de esos mismos estratos. La ilegitimidad parece ser aceptada en estos niveles. No obstante, es más factible que las mujeres que ascienden socialmente constituyan familias y también que requieran menor tiempo para formarlas. ${ }^{25}$ Las mujeres parecen estar más protegidas por sus padres cuando son jóvenes, y estas últimas tienen ma yor posibilidad de establecer familias en menor tiempo. Sin embargo, tanto la religión como la religiosidad no logran buenas predicciones. Esto puede deberse a que las enseñanzas de la Iglesia no llegan a ser lo suficientemente profundas como para evitar las relaciones sexuales antes del matrimonio. Por otra parte, estos hechos indican que simplemente el credo religioso y la asistencia a la iglesia son malas mediciones de la religión y de la religiosidad.

Por último, la presente investigación indica que las comparaciones culturales cruzadas pueden arrojar luz sobre el tópico de la ilegitimidad y el matrimonio subsecuente. Tales investigaciones, relativas primordialmente al matrimonio después de la ilegitimidad, han sido descuidadas de alguna manera, y deberán hacerse más estudios en lo futuro. Otras investigaciones en Brasil sobre el noviazgo, las uniones, la anticoncepción, el embarazo y el aborto parecen esenciales para una mejor comprensión de todo el proceso.

25 Cf. Goode, op. cit. pp. 25 y 29, donde el autor indica que las parejas socialmente ascendentes cambian esa relación consensual y pasan a formar una pareja formalmente casada. 